\title{
Qualitative and Quantitative Evaluation of EEG Signals in Epileptic Seizure Recognition
}

\author{
S. A. Hosseini \\ Dept. of Electrical Engineering, Center of Excellence on Soft Computing and Intelligent Information Processing, \\ Ferdowsi University of Mashhad, Mashhad, Iran \\ E-mail: hosseyni@kiaeee.org \\ M-R. Akbarzadeh-T \\ Dept. of Electrical and Computer Engineering, Center of Excellence on Soft Computing and Intelligent Information \\ Processing, Ferdowsi University of Mashhad, Mashhad, Iran
}

M-B. Naghibi-Sistani

Dept. of Electrical and Biomedical Engineering, Ferdowsi University of Mashhad, Mashhad, Iran

\begin{abstract}
A chaos-ANFIS approach is presented for analysis of EEG signals for epileptic seizure recognition. The non-linear dynamics of the original EEGs are quantified in the form of the hurst exponent $(H)$ and largest lyapunov exponent $(\lambda)$. The process of EEG analysis consists of two phases, namely the qualitative and quantitative analysis. The classification ability of the $\mathrm{H}$ and $\lambda$ measures is tested using ANFIS classifier. This method is evaluated with using a benchmark EEG dataset, and qualitative and quantitative results are presented. Our inter-ictal EEG based diagnostic approach achieves $97.4 \%$ accuracy with using 4-fold cross validation. Diagnosis based on ictal data is also tested in ANFIS classifier, reaching 96.9\% accuracy. Therefore, our method can be successfully applied to both inter-ictal and ictal data.
\end{abstract}

Index Terms - ANFIS, EEG, Hurst Exponent, Lyapunov Exponent

\section{Introduction}

Epilepsy is a brain disorder that is characterized by sudden and recurrent seizures. Epilepsy can cause abnormal electrical activity in the brain and may alter consciousness, perception, sensation, behavior and body movement. Patients experience varied sympto ms during seizures depending on the location and extent of the affected brain tissue. Most seizures are very brief and is rarely life threatening. Depending on the extent of the involvement of brain areas during the epilepsy, it can be divided into two main types that according to the International League Against Epilepsy (ILAE), in 1981, includes: 1- Generalized seizures that involve almost the entire brain, 2- Partial (or focal) seizures that originate from a circumscribed area of the brain and remain restricted to that area [1]. Generalized seizures can be divided into several main types, such as Absence, Atypical Absence, Myoclonic, Clonic, Tonic, Tonicclonic, and Atonic seizures. In addition, Partial seizures can be divided into three main types such as Simple partial, Complex partial, and Secondarily generalized seizures.

Due to a large number of patients in intensive care units (ICU) and the need for continuous observation of such conditions, several methods for epileptic seizure recognition have been developed in the past. Several quantitative system approaches incorporating statistical techniques, dynamical systems and optimization for brain disorders [2]. In assessment of epilepsy, brain activity plays a central role. Electroencephalography (EEG) is a technique, which contains much information about the patient's psycho-physiological state [3]. Therefore, EEG has become the premier diagnostic method for epilepsy recognition. EEG can be recorded in two essential ways: The first and most common is non-invasive recording known as scalp recording. The second is invasive recording that often is known as inter-cranial EEG. Frequency bands of EEG signals are interesting to be interpreted such as delta $(1-4 \mathrm{~Hz})$, theta (4-8 Hz), alpha $(8-13 \mathrm{~Hz})$, beta $(13-30 \mathrm{~Hz})$ and gamma (> $30 \mathrm{~Hz})$.

In principle, there are two different scenarios of how a seizure could evolve. It could be caused by a sudden and abrupt transition, in which case it would not be preceded by detectable dynamical changes in the EEG. Such a scenario would be conceivable for the initiation of seizures in primary generalized epilepsy. Alternatively, this transition could be a gradual change in dynamics, which could in theory, is detectable. This type of transition could be more likely in focal epilepsies. About dynamical states of epileptic EEG signals, there are some main classic states of inter-ictal, pre-ictal, ictal, and post-ictal; but clinical and laboratory experiments leave little doubt that a pre-seizure period 
exists in temporal lobe and perhaps other forms of epilepsy. Its existence, however, raises fundamental questions about what constitutes a seizure, what brain regions are involved in seizure generation, and whether discrete inter-ictal, pre-ictal, ictal, and post-ictal physiologies exist, or blend together in a continuous process [4].

Feature extraction process plays a very important role on the classification performance. In this research, Nonlinear measures like Correlation dimension, Fractal dimension, Hurst exponent and Lyapunov exponent, quantify the degree of complexity in a time series. Features are selected so that they capture the differences between the epileptic and normal EEG. Fuzzy set theory plays an important role in dealing with uncertainty when making decisions in medical applications. Fuzzy sets have attracted the growing attention and interest in modern information technology, production technique, decision making, pattern recognition, diagnostics, and data analysis. Neuro-fuzzy systems are fuzzy systems, which use Artificial Neural Networks (ANNs) theory in order to determine their properties (fuzzy sets \& fuzzy rules) by processing data.

A lot of research has been undertaken in assessment of epilepsy over the last few years. Gotman [5] presented a computerized system for recognizing a variety of seizures, in year 1982. Murro et al. [6] developed a seizure recognition system based on the discriminant analysis of the EEG signal recorded from the intracranial electrodes. Choosing suitable features is important for seizure recognition. Many features have been investigated based on time domain and frequency domain [7,8], wavelet transformation [9,10], Fourier Transformation [11], energy distribution in timefrequency plane [12], and chaotic features [13-17]. Subasi [9] used a method for analysis of EEG signals using discrete wavelet transform (DWT) and classification using an ANFIS. Non-linear features of EEG signals have also often explained complex structure of epilepsy $[18,19]$. Kannathal et al. [16] have shown the importance of various entropies for recognition of epilepsy. Adeli et al. [20] presented a wavelet-chaos methodology for analysis of EEGs and delta, theta, alpha, beta, and gamma sub-bands of EEGs for recognition of seizure. Ghosh-Dastidar et al. [21] presented a wavelet-chaos-neural network methodology for classification of EEGs into healthy, ictal, and interictal EEGs.

From studies reported in the literature, EEG signals can be considered chaotic. In this research, an approach based on Adaptive Neuro-Fuzzy Inference System (ANFIS) was presented for the seizure recognition. The main aim of this research is to produce a multi-aspect combination of chaos and ANFIS for epileptic seizu re recognition using EEG signals.

\section{Methods and Materials}

\subsection{Database}

For studying epilepsy, the subjects that are used are either human o animal. EEG signals used for this research are obtained from Bonn University, Germany, which is available in public domain [22]. The complete datasets consists of five sets of data (denoted $A-E$ ), each containing 100 single-channel EEG segments. Each segment has $N=4096$ sampling points over 23.6 seconds. All EEG signals were recorded with the same 128-channel amplifier system and 12 b it $A / D$ resolution, which the sampling rate of the data was $173.61 \mathrm{~Hz}$, which it have the spectral bandwidth of the acquisition system is between 0.5 to $85 \mathrm{~Hz}$. These segments were selected and cut out from continuous multi-channel EEG recordings after $v$ isual inspection for artifacts such as muscle activity, eye movements etc. Sets $A$ and $B$ have been recorded from external surface EEG recordings of five healthy volunteers in the wake state with eyes open and eyes closed, respectively. Sets $C, D$ and $E$ have been recorded from depth electrodes EEG recordings from five patients. Sets $A$ and $B$ have been recorded during normal state. Set $D$ were recorded from within the epileptogenic zone (Inter-ictal period), and those in set $C$ from the hippocampal formation of the opposite hemisphere of the brain (Inter-ictal period). Set $E$ has been recorded during seizure activity (Ictal). Figure 1 shows the sample recordings of EEG signals obtained from five sets $A-E$. For a more detailed description of the data, please refer to the [22].

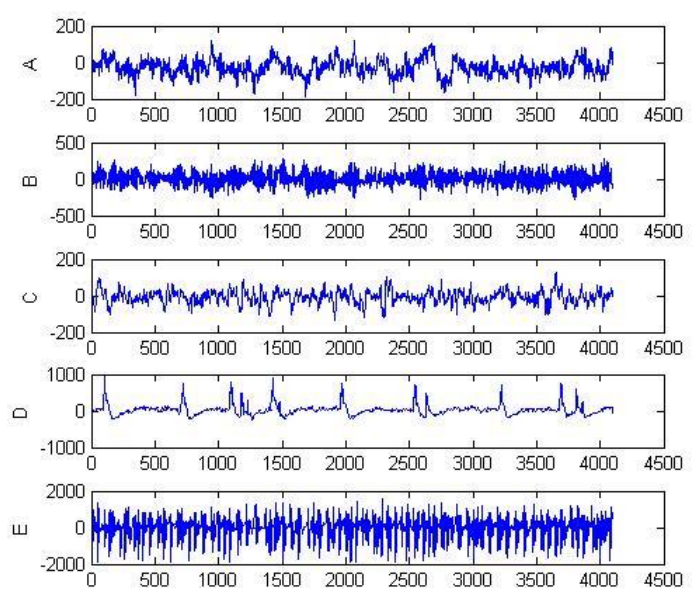

Fig. 1: Sample recordings of $A, B, C, D$ and $E$ dataset from top to bottom

\subsection{Features Extraction}

Choice of the methods and measures for discriminating dynamical changes of epileptic signals highly depends on the characteristics of epileptic EEG signals, on the other hand, regarding type of seizure, specifications of individuals who data are recorded from them etc, are important parameters that have significant effect on results of recognition and classifying of epileptic EEG signals. 
In this research to concentrate on the recognition of dynamic changes of EEG signals recording, a short epoch of data is selected. The time series of data are divided into 2 seconds epochs with an overlap of 1 second.

Non-linear and chaotic measures have received the most attention in comparison with the measures mentioned before such as time domain, frequency domain, time-frequency etc.

\section{1) Hurst exponent}

The name Hurst exponent $(\mathrm{H})$ derives from Harold Edwin Hurst in 1951 [23]. The Hurst exponent is directly related to the Fractal dimension. a s mall Hurst exponent has a higher fractal dimension and vice versa. Brownian walks can be generated from a defined Hurst exponent. $H$ has a value between 0 and $1(0 \leq H \leq 1)$. If a value $H$ in the range $0.5<H<1$, the random walk will be a long-term memory process (or positive correlated time series or persistent). A value $H$ in the range $0<H<0.5$ can indicate anti-correlated time series (anti-persistent). A value of $H=0.5$ can indicate a completely uncorrelated time series.

Hurst exponent are shown in Figures 2, 3 and 4 corresponding to the $A, C$ and $E$ data, respectively.

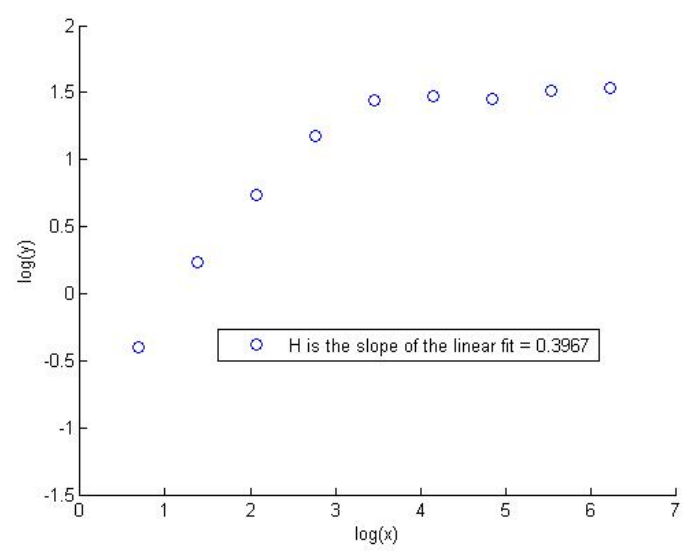

Fig. 2: Hurst exponent of the EEG signal corresponding to normal time series $(A)$.

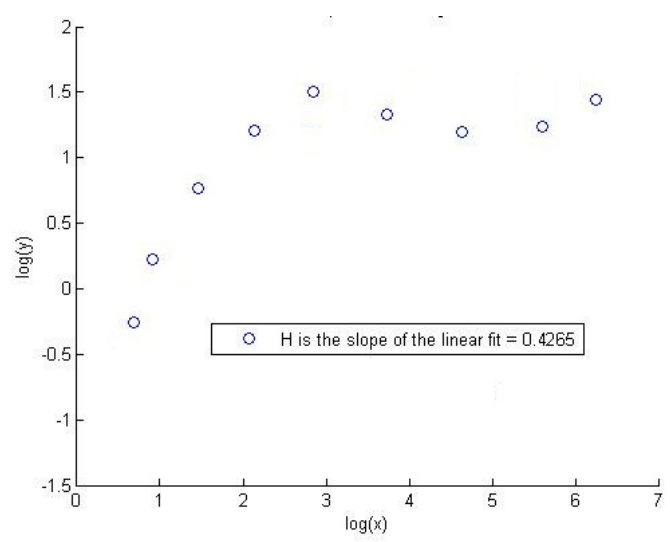

Fig. 3: Hurst exponent of theEEG signal corresponding to inter-ictal time series $(C)$.

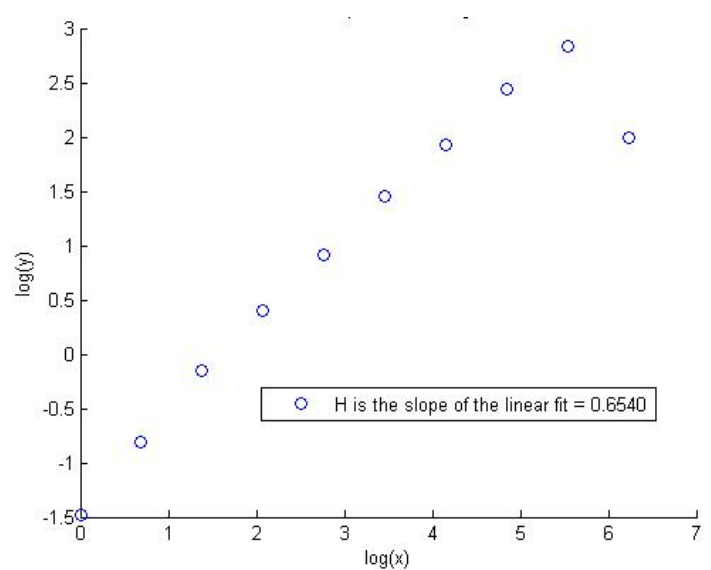

Fig. 4: Hurst exponent of theEEG signal corresponding to ictal time series $(E)$.

From the above Figures 2, 3 and 4 it is clear that the value of Hurst exponent for non-epileptic signal sets $A$, $B$ is less than epileptic signal set $E$.

\section{2) Lyapunov exponent}

The lyapunov exponents have been proven the useful dynamical quantity for the chaotic system analysis [25]. Lyapunov exponents define the average exponential rates of divergence or convergence of the nearby orbits in the phase space and can be estimated using largest lyapunov exponent $(\lambda)$.

A negative lyapunov exponent indicates that, the orbit attracts to a stable fixed point (or stable periodic orbit). A lyapunov exponent of zero indicates that, the orbit is a neutral fixed point (or an eventually fixed point). A positive lyapunov exponent indicates that, the orbit is unstable and chaotic. Lyapunov exponent is shown in Figure 5 corresponding to the $A, C$ and $E$ data, respectively.

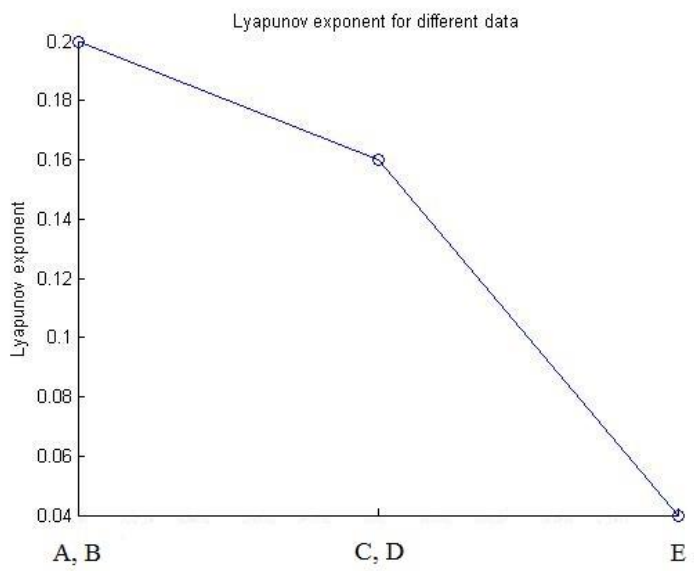

Fig. 5: Plot of $\lambda$ values for EEG signals between three different classes.

From the above Figure, it is clear that the value of Lyapunov exponent for epileptic signal set $E$, is less than non-epileptic signal sets $A, B$. 


\subsection{Classification}

The Adaptive Neuro-Fuzzy Inference System, first introduced by Jang in 1993 [24,25]. The ANFIS is a fuzzy Sugeno model put in the framework of adaptive systems to facilitate learning and adaptation [24]. Jang showed that even if human expertise is not available it is possible to intuitively set up practical membership function and employs the neural training process to generate a set of fuzzy IF-THEN rules that Approximate a desired data set [24]. In this structure, there are five layers in the overall network framework, including the fuzzification layer, product layer, normalization layer, defuzzification layer and the total output layer.

\section{Computation Results and Analysis}

The process of EEG analysis consists of two states: first the qualitative analysis and second the quantitative analysis of EEG signals.

The qualitative results are presented in this section. The value of Hurst exponent for non-epileptic signal sets $A, B$ is less than epileptic signal set $E$. In addition, the value of Lyapunov exponent for epileptic signal set $E$, is less than non-epileptic signal sets $A, B$. The reduce in $\lambda$ value, and the increase in $H$ value to mean the reduction in brain system complexity for subjects with seizure state, therefore the number of the necessary dynamic equations for the description of the brain state in the seizure state decreases.

The quantitative results and comparison with the other researchers presented in this section. As shown in Table. 1, we designed three experiments to test the ability of our classifier to separate: 1 - Normal EEG (sets $A$ and $B$ ) vs. inter-ictal EEG (sets $C$ and $D$ ) 2- Normal EEG (sets $A$ and $B$ ) vs. ictal EEG (set $E$ ) 3- Inter-ictal EEG (sets $C$ and $D$ ) vs. ictal EEG (set $E$ )

Table 1: The classification accuracy for three experiments

\begin{tabular}{|c|c|c|}
\hline Number & Experiment & Classification accuracy (\%) \\
\hline 1 & Normal vs. inter-ictal & 97.4 \\
\hline 2 & Normal vs. ictal & 96.9 \\
\hline 3 & Inter-ictal vs. ictal & 96.5 \\
\hline
\end{tabular}

The classifier is validated using 4-fold cross validation on 400, 300 and 300 samples, respectively, in experiments 1, 2 and 3 .

In experiment 1 , the accuracy using inter-ictal EEG for epileptic recognition reaches $97.4 \%$. In experiment 2 , the accuracy using ictal data reaches 96.9\%. In experiment 3, 96.5\% accuracy shows our system can distinguish ictal vs. inter-ictal EEG.

For $A-E$ or $A, B-E$ classification, the result obtained from our method is better than [16] and [9] researches with $7.4 \%$ and $3.4 \%$ difference to discriminate EEGs, respectively. For $A, B, C, D-E$ classification, the accuracy obtained from [1] is better than [12] with $0.04 \%$ difference.

\section{Conclusion}

This research has presented the analysis of the EEG signals for the characterization of the epileptic behavior of the brain activity. We investigated the chaotic dynamics underlying the EEG in patients with Epilepsy's disease by non-linear analysis to understand the role of chaos in brain function. Therefore, presents a novel method for epileptic seizure recognition using chaotic features and ANFIS classifier. We calculated the hurst exponent and largest lyapunov exponent for non-linear analysis of EEG signals. The process of EEG analysis consists of two states: first the qualitative analysis and second the quantitative analysis of EEG signals. The reduce in $\lambda$ value and the increase in $H$ value to mean the reduction in brain system complexity for subjects with seizure state, therefore the number of the necessary dynamic equations for the description of the brain state in the seizure state decreases. Therefore, chaotic features could be helpful to distinguishing between epileptic EEG and normal EEG. This method is evaluated using a benchmark EEG dataset, and qualitative and quantitative results are presented. Our inter-ictal EEG based diagnostic approach achieves 97.4\% accuracy in 4-fold cross validation. Diagnosis based on ictal data is also tested in ANFIS classifier, reaching $96.9 \%$ accuracy. Therefore, our method can be successfully applied to both inter-ictal and ictal data. We show that non-linear analysis can provide a promising tool for detecting relative changes in the complexity of brain dynamics, which may not be detected by conventional linear analysis. The testing performance of the model diagnostic system is found to be satisfactory and we think that this system can be used in clinical studies in the future after it is developed.

\section{Acknowledgments}

The authors would like to acknowledge Dr. R.G. Andrzejak of Bonn University, Germany, for providing permission to use the EEG signals available in the public domain.

\section{References}

[1] L. Guo, D. Rivero, J. Dorado, J. R. Rabũnal, and A. Pazos, "Automatic epileptic seizure detection in EEGs based on line length feature and artificial neural networks," Journal of Neuroscience Methods, vol. 191, no. 1, pp. 101-109, 2010.

[2] W. Chaovalitwongse, P. Pardalos, L. D. Iasemidis, D. S. Shiau, and J. C. Sackellares, "Dynamical 
approaches and multi-quadratic integer programming for seizu re pred iction," Optimization methods and Software, vol. 20, no. 2-3, pp. 389400, 2005.

[3] Y. Kumar, and M. L. Dewal, "Complexity Measures for Normal and Epileptic EEG Signals using ApEn, SampEn and SEN," International Journal of Computer \& Communication Technology (IJCCT), vol. 2, Issue 7, pp. 6-12, 2011.

[4] B. Litt, and K. Lehnertz, "Seizure prediction and the preseizure period," Current Opinion in Neurology, vol. 15, no. 2, pp. 173-177, 2002.

[5] J. Gotman, "Automatic recognition of epileptic seizures in the EEG," Electroencephalogr Clin Neurophysiol, vol. 54, pp. 530-540, 1982.

[6] A. M. Murro, D. W. King, J. R. Smith, B. B. Gallagher, H. F. Flanigin, and K. Meador, "Computerized seizure detection of complex partial seizures," Electroencephalogr Clin Neurophysiol, vol. 79, pp. 330-333, 1991.

[7] V. Srinivasan, C. Eswaran, and N. Sriraam, "Artificial Neural Network Based Epileptic Detection Using Time-Domain and FrequencyDomain Features," Journal of Medical Systems, vol. 29, no. 6, pp. 647-660, 2005.

[8] V. P. Nigam, and D. Graupe, "A neural-networkbased detection of epilepsy," Neurol Res, vol. 26, no. 1, pp. 55-60, 2004.

[9] A. Subasi, “Application of adaptive neuro-fuzzy inference system for epileptic seizure detection using wavelet feature extraction," International Journal of Computers in Biology and Medicine, vol. 37, pp. 227-244, 2007.

[10] I. Gu"ler, and E. D. U"beyli, "Application of adaptive neuro-fuzzy inference system for detection of electrocardiographic changes in patients with partial epilepsy using feature extraction," International Journal of Expert Systems with Applications, vol. 27, pp. 323-330, 2004.

[11] P. Y. Ktonas, "Automated spike and sharp wave (SSW) detection," In: Gevins A.S., \& Remond, A., (Ed.), Methods of analysis of brain electrical and magnetic signals, Amsterdam: Elsevier, pp. 211241, 1987.

[12] A. Tzallas, M. Tsipouras, and D. Fotiadis, "Automatic seizure detection based on timefrequency analysis and artificial neural networks," Computational Intelligence and Neuroscience, pp. 1-13, 2007.

[13] D. E. Lerner, "Monitoring changing dynamics with correlation integrals: Case study of an epileptic seizure," Physica D, vol. 97, no. 4, pp. 563-576, 1996.
[14] M. Tito, M. Cabrerizo, M. Ayala, A. Barreto, I. Miller, P. Jayakar, and M. Adjouadi "Classification of electroencephalographic seizure recordings into ictal and interictal files using correlation sum," International Journal of Computers in Biology and Medicine, vol. 39, pp. 604-614, 2009.

[15] V. Srinivasan, C. Eswaran, and N. Sriraam, "Approximate Entropy-Based Epileptic EEG Detection Using Artificial Neural Networks," IEEE Transactions on information Technology in Biomedicine, vol. 11, no. 3, 2007.

[16] N. Kannathal, C. M. Lim, U. Rajendra Acharya, and P. K. Sadasivan, "Entropies for detection of epilepsy in EEG," International Journal of Computer Methods and Programs in Biomedicine, vol. 80, no. 3, pp. 187-194, 2005.

[17] S. Pravin-Kumar, N. Sriraam, P. G. Benakop, and B. C. Jinaga, "Entropies Based Detection of Epileptic Seizures with Artificial Neural Network Classifiers," International Journal of Expert systems with application, vol. 37, pp. 3284-3291, 2010.

[18] H. Ocak, "Automatic detection of epileptic seizures in EEG using discrete wavelet transform and approximate entropy," International Journal of Expert Systems with Applications, vol. 36, pp. 2027-2036, 2009.

[19] D. L. Iasemidis and J. C. Sackellares, "Chaos Theory and Epilepsy," The NeuroScientist, vol. 2, no. 2, pp. 118-126, 1996.

[20] H. Adeli, S. Ghosh-Dastidar and N. Dadmehr, "A Wavelet-Chaos Methodology for Analysis of EEGs and EEG Subbands to Detect Seizure and Epilepsy," IEEE Transactions on Biomedical Engineering, vol. 54, no. 2, 2007.

[21] S. Ghosh-Dastidar, H. Adeli, and N. Dadmehr, "Mixed-Band Wavelet-Chaos-Neural Network Methodology for Epilepsy and Epileptic Seizure Detection," IEEE Transactions on Biomedical Engineering, vol. 54, no. 9, 2007.

[22] R. G. Andrzejak, K. Lehnertz, F. Mormann, C. Rieke, P. David, and C. E. Elger. (2001). Indications of nonlinear deterministic and finite dimensional structures in time series of brain electrical activity: Dependence on recording region and brain state. Physical Review E, vol. 64, Available: http://epileptologie-bonn.de

[23] H. E. Hurst, "Long-term storage of reservoirs: an experimental study," Transactions of the American society of civil engineers, vol. 116, pp. 770-799, 1951.

[24] J.-S.R., Jang, and C. T. Sun, "Neuro Fuzzy Modeling and Control," Proceedings of the IEEE Transactions, vol. 83, no. 3, pp. 378-406, 1995. 
[25] Jang, J.-S.R., “ANFIS: Adaptive-network-based fuzzy inference system," IEEE Transactions Systems, Man and Cybernetics, vol. 23, no. 3, pp. 665-685, 1993.

\section{Authors' Profiles}

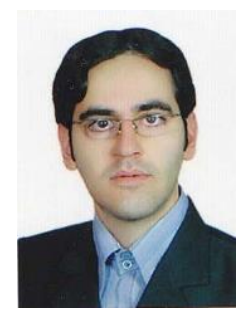

Seyyed Abed Hosseini $\mathrm{He}$ is currently Ph.D. candidate at the Control Engineering of the Ferdowsi University of Mashhad, Iran. He currently holds as instructor in the dept. of electrical engineering at Islamic Azad University Shahrood Branch.

$\mathrm{He}$ is a member of the Iranian Society for Biomedical Engineering, Tehran, Iran. He has published over 30 journal and conference papers. $\mathrm{He}$ is currently a Reviewer for international journals and conferences. He participated in international conferences as a session chair.

His research interests include recognition of emotional stress states based on the analysis of EEG and psychophysiological signals in order to improve human-computer interaction, functional brain modeling, non-linear and chaotic analysis, fuzzy-neural networks, and digital design with FPGA and CPLD.

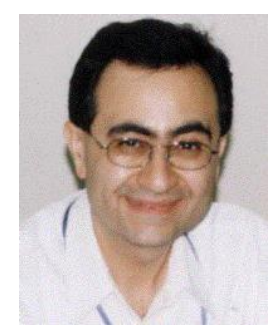

Mohammad-R. Akbarzadeh-T. (Senior Member, IEEE) received his Ph.D. on Evolutionary Optimization and Fuzzy Control of Complex Systems from the department of electrical and computer engineering at the University of New Mexico in 1998.

He currently holds dual appointment as professor in the departments of electrical engineering and computer engineering at Ferdowsi University of Mashhad. In 2006-2007, he completed a one-year visiting scholar position at Berkeley Initiative on Soft Computing (BISC), UC Berkeley. From 1996-2002, he was affiliated with the NASA Center for Autonomous Control Engineering at University of New Mexico (UNM).

Dr. Akbarzadeh is the founding president of the Intelligent Systems Scientific Society of Iran, the founding councilor representing the Iranian Coalition on Soft Computing in IFSA, and a council member of the Iranian Fuzzy Systems Society. He has received several awards including: the IDB Excellent Leadership Award in 2010, The IDB Excellent Performance Award in 2009, the Outstanding Faculty Award in 2008 and 2002, the IDB Merit Scholarship for High Technology in 2006, the Outstanding Faculty Award in Support of Student Scientific Activities in 2004, Outstanding
Graduate Student Award in 1998, and Service Award from the Mathematics Honor Society in 1989.

His research interests are in the areas of evolutionary algorithms, fuzzy logic and control, soft computing, multi-agent systems, complex systems, robotics, and biomedical engineering systems. He has published over 250 peer-reviewed articles in these and related research fields.

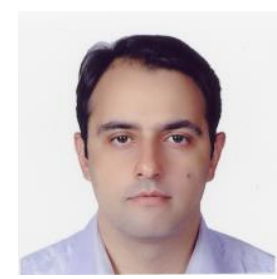

Mohammad-Bagher NaghibiSistani He received the B.S. degree in electronics from the University of Tehran, Tehran, Iran, in 1991, the M.S. degree at control engineering from the University of Tehran, Tehran, Iran, in 1995, and the Ph.D. degree in control engineering from the Ferdowsi University of Mashhad, Iran, in 2005.

He currently is Assistant Professor at the Department of Electrical Engineering and Biomedical Engineering, Ferdowsi University of Mashhad.

His research interests include reinforcement learning, soft computing, optimal control, and Machine learn ing. He has published over 50 journal and conference papers.

How to cite this paper: S. A. Hosseini, M-R. Akbarzadeh-T, M-B. Naghibi-Sistani,"Qualitative and Quantitative Evaluation of EEG Signals in Epileptic Seizure Recognition", International Journal of Intelligent Systems and Applications(IJISA), vol.5, no.6, pp.41-46, 2013.DOI: 10.5815/ijisa.2013.06.05 